\title{
Vortex shedding from a wind turbine blade section at high angles of attack.
}

\author{
Alberto Pellegrino, Craig Meskell \\ School of Engineering, Trinity College Dublin, Dublin 2, Ireland \\ E-mail addresses: pellega@tcd.it (A. Pellegrino),cmeskell@tcd.ie (C. Meskell).
}

\begin{abstract}
The unsteady flow around a stationary two-dimensional wind turbine blade section (NREL S809) has been simulated using unsteady RANS with the SST turbulence model at $\operatorname{Re}=10^{6}$ and high angles of attack. Vortex shedding frequency non-dimensionalised by the projected area of the blade is in the range $0.11<S t<0.16$. The fluctuating coefficients at the harmonics of the fundamental vortex shedding frequency for lift, drag and pitching moment are presented. The lift force and the pitching moment (calculated with respect to the midchord) are dominated by the fundamental vortex shedding frequency, but significant contributions at the higher harmonics are also present. For the drag the second harmonic is as significant as the fundamental frequency, with the third and fourth harmonics contributing to a lesser extent. From the results, it is inferred that the camber of the airfoil does not substantially affect the force coefficients, suggesting that the results presented may be generally applicable to turbine blades of different geometry.
\end{abstract}

Keywords: Wind turbine blade, Vortex shedding, High angles of attack, Strouhal number.

Citation: Pellegrino, A., Meskell, C., 2013. Vortex shedding from a wind turbine blade section at high angles of attack. Journal of Wind Engineering and Industrial Aerodynamics 121, 131-137.

http://dx.doi.org/10.1016/j.jweia.2013.08.002 


\section{Introduction}

In 2012, there were a total of 284 GW of wind generating capacity installed globally (Anon, 2013). The majority of the machines installed are horizontal axis design (HAWT). As with any technology that harvests ambient energy, wind turbines must be permanently exposed to environmental extremes. As a result, the minimum design requirement for wind turbines as defined by the IEC (61400-1:2005+A1:2010) include specifications for extreme wind loading conditions (see section 6.3 of the standard). For example, the structure must be designed to withstand steady, turbulent and deterministic gusts which occur on average only every 50 years. As part of the transient wind environment a sudden change of wind direction of up to $180^{\circ}$ must be considered. Consider a turbine blade, which will have either been feathered towards the prevailing mean wind in severe wind conditions, or will be at a low angle of attack relative to the local flow direction in operational conditions. In the extreme change of direction scenario, the blade will be suddenly inclined at high angle of attack to the local flow. Hence the section will behave more like a bluff body than a streamlined airfoil.

The problem of bluff body flow is well known and widely studied. A review on this problem is presented in Roshko (1993). Furthermore, vortex shedding induced vibration (VIV) can be a problem for a wide range of structures exposed to a cross flow since the resulting vibration amplitudes can lead to catastrophic failure. In particular VIV can be expected in flexible slender bodies of any cross-section. The literature is rich in comprehensive reviews on VIV (e.g. Williamson and Govardhan, 2008; Sarpkaya, 2004; Blevins, 1990). A clear presentation of the problem of VIV for a generic bluff body can be found in a recent book by Paidoussis et al. (2010).

As fluctuations of vorticity develop in the unstable wake, periodic forces and moments result on the body. The fluctuating lift and drag are dominated by the actions from the vortex shedding, and are due mainly to the fluctuating pressures acting on the surface of the cylinder. The lift fluctuation energy is concentrated in a band around $f_{v}$, whereas the drag fluctuation energy is concentrated in a band around $2 f_{v}$ (Sonneville, 1976; Farell, 1981). For the case of a vibrating solid body, when the vortex shedding frequency approaches the moving body frequency, synchronization takes place and the structure undergoes potential and unwanted vortex induced vibration. This is the so called "lock-in" phenomenon.

Most studies of vortex shedding to date have been carried out on the 
circular cylinder body due to its simple geometric shape. For other shapes, such as rectangular prisms, long-span bridge sections or blade section, there is limited knowledge about vortex shedding, even for many problems of practical importance. Some studies have been conducted on inclined flat plates (e.g. Fage and Johansen, 1927; Perry and Steiner, 1987) and elliptic bodies (e.g. Nair and Sengupta, 1996, 1997), the cross-flow behaviour of which, at high angles of attack, can give a good idea of what could happen in the case of a blade section.

There are few studies on bluff bodies at high Reynolds number. Moreover very few results can be found in the literature regarding vortex shedding from airfoil sections. Experiments have been conducted for example by Medici and Alfredsson (2006). They reported that the Strouhal number $(S t \approx 0.12)$ from the rotor disc is comparable with that reported for a solid disc. However, it is worth noting that this Strouhal number is also consistent with slender structural components at high angles (Simiu and Scanlan, 1978).

While the ultimate objective is to develop what Paidoussis et al. (2010) call a "coupled system model" of vortex induced vibration for wind turbine blades which can be used as part of a design or certification process, the current work represents an initial step towards this goal. This corresponds to the "forced system model" described by Paidoussis et al. (2010). The focus here is on the behavior of the unsteady wake behind the fixed airfoil at high angles of attack, which acts as a bluff body.

\section{Methodology}

A series of two-dimensional unsteady simulations of the flow around the NREL S809 airfoil at different angles of attack were performed. Figure 1 shows the S809 with the positive angular direction specified for the airfoil inclination with respect to the freestream direction. Two typical configurations are illustrated: airfoil with the sharp trailing edge (STE) in down-wind position, Figure 1(a), and up-wind position, Figure 1(b). All calculations have been made at $R e=10^{6}$. The software used is the commercially available URANS solver ANSYS-Fluent ${ }^{\circledR}$.

In Section 3.1 details of the fluid-dynamic model validation process are given. The validation concerns both the near field and the far field, keeping the same flow properties and Reynolds number, to fully test the fluid-dynamic model. For the near field validation, a steady-state analysis has been conducted (range $\alpha=\left[0^{\circ} \rightarrow 18^{\circ}\right]$ ). Such analysis deals with the capability of 
the model to capture the physics in the near vicinity of the airfoil. For the far field instead, where the vortex shedding phenomenon develops, the standard case of unsteady flow around a circular cylinder was analysed, as there is almost no detailed experimental data available for vortex shedding from airfoils at high angle of attack and high Reynolds number. The unsteady vortex shedding analysis requests accuracy in the far field behind the body too.

Once verified, the fluid-dynamic model (at the same Reynolds number and using the same meshing density and turbulence model) has been used to perform simulations of unsteady the flow around the wind turbine blade section at different angles of attack, in the whole positive range $\alpha=\left[0^{\circ} \rightarrow\right.$ $\left.180^{\circ}\right]$ and negative range $\alpha=\left[0^{\circ} \rightarrow-180^{\circ}\right]$, stepping by $10^{\circ}$, to give a clear idea of the global airfoil behaviour.

Using the data obtained the non-dimensional shedding frequency (i.e. the Strouhal number) and non-dimensional loading coefficients have been calculated for each angle of attack.

\subsection{Fluid-Dynamic Model}

The fluid domain, illustrated in Figure 2 has been created with the ANSYS ${ }^{\circledR}$ default meshing tool. The domain extends to $10 c$ (airfoil chord) length from the body in the upstream direction, $40 c$ in the downstream direction and $20 \mathrm{c}$ in the cross-flow direction. The reference chord length is $1 \mathrm{~m}$.

The fluid domain has been divided into different zones in which the mesh density is different, keeping it finer in the vicinity of the airfoil and fading in the far wake downstream. The inner zone has been modelled as a circle in order to facilitate the rotation of the airfoil with respect to the incoming flow. The mesh is unstructured, with triangular elements throughout except the quadratic elements in the near-wall region. The near wall mesh has 512 cells in the chord-wise direction and the overall mesh is composed in total of approximately 100,000 nodes.

Turbulence closure is modeled by the Shear Stress Transport (SST) eddy viscosity model. This transition turbulence model requires a very fine mesh in the vicinity of the airfoil. For that purpose, the height of the first cell has been tuned in order to obtain a $y^{+}$value below 2 everywhere on the airfoil surface.

The airfoil throughout was specified by no-slip conditions. Inlet flow was specified at the upstream boundary of the domain, with the following inflow 
conditions shown in Table 1. Flow speed has been calculated starting from the $R e$ value of $10^{6}$.

\begin{tabular}{llll}
\hline Inlet flow properties $\left(R e=10^{6}\right)$ & \\
\hline & & & $\mathrm{m} / \mathrm{s}$ \\
Velocity & $U_{\infty}$ & 14.607 & $\mathrm{~kg} / \mathrm{m}^{3}$ \\
Density & $\rho$ & 1.225 & $\mathrm{~kg} / \mathrm{ms}$ \\
Viscosity & $\mu$ & $1.7894 \times 10^{-5}$ & \\
\hline
\end{tabular}

Table 1: Inlet flow properties.

Outlet condition was specified in the downstream boundary of the domain with a reference pressure $p_{\text {gauge }}=0$.

Computations have been made using the PISO scheme, with Second Order Upwind discretisation for the momentum and a time-step of $0.005 \mathrm{~s}$.

\subsection{Harmonic description of forces}

The lift force, $L$, is defined perpendicular to the flow direction, while the drag is parallel to the flow. When considering the loads on airfoils it is common to use the pitching moment, $M$, around the quarter chord (i.e. a point on the chord line, $25 \%$ of the chord from the leading edge) as this is theoretically the aerodynamic centre. However, as this airfoil is at such a high angle of attack and the behaviour as a bluff body is of most interest here, the pitching moment has been taken through the mid-chord point (i.e. equidistant from leading and trailing edge).

The aerodynamic loads can be non-dimensionalised using the chord as the reference length:

$$
\begin{aligned}
C_{l} & =\frac{L}{0.5 \rho U_{\infty}^{2} c} \\
C_{d} & =\frac{D}{0.5 \rho U_{\infty}^{2} c} \\
C_{m} & =\frac{M}{0.5 \rho U_{\infty}^{2} c^{2}}
\end{aligned}
$$

The time series of the aerodynamic forces and moment coefficients have been decomposed into Fourier series, as shown in Eq. (2), in order to quantify the vortex induced excitation at higher frequencies. 


$$
C_{g}(t)=C_{g 0}+\sum_{i} C_{g i} \sin \left(i 2 \pi f_{v} t+\phi_{i}\right)
$$

where $C_{g}$ is the overall instantaneous force coefficient, $C_{g 0}$ is the mean coefficient, $C_{g i}$ is the amplitude of the fluctuating force coefficient for the $i^{\text {th }}$ harmonic, $f_{v}$ is the the vortex shedding frequency, $\phi_{i}$ is the phase for the $i^{\text {th }}$ harmonic.

Once the fundamental vortex shedding frequency has been obtained for each angle of attack, the Fourier coefficients at harmonics of this frequency are obtained. For the lift force, the first three harmonics are needed to fit the time data, whereas the first four harmonics are required for the drag force and the pitching moment.

The fundamental vortex shedding frequency $f_{v}$ can be normalised using the characteristic length $(d)$ and the local freestream speed $\left(U_{\infty}\right)$ as is usually done for bluff bodies, resulting in the non-dimensional frequency (Strouhal number) defined as:

$$
S t=\frac{f_{v} d}{U_{\infty}}
$$

The choice of the characteristic length will be considered below.

\section{Results and discussions}

\subsection{Validation}

Limited data are available for vortex shedding from airfoils at high angle of attack. Therefore, in order to have confidence in the modeling approach, at first it was sensible to conduct a preliminary unsteady analysis on a circular cylinder for which detailed experimental results are present. For this test case the same model setting and conditions are used, as well as the characteristic length (cylinder diameter, $d=1 \mathrm{~m}$ ). In Table 2 experimental and current simulated values of Strouhal number, lift coefficient root mean square and mean drag coefficient are given. Results show a good agreement with experiments for both the vortex shedding frequency and the amplitude of the aerodynamic forces. It must be said that the model needed to be tuned: reliable results required a residuals tolerance of at least $10^{-5}$; in addition, a momentum under-relaxation factor value of 0.5 (the solver default value was 0.7) is needed to avoid residual divergence at the beginning of the simulation 
due to the high turbulence level associated with the relatively high Reynolds number $\left(R e=10^{6}\right)$.

In Figure 3 instead the lift and drag curves at steady-state conditions are presented. The current results are compared with Delft University of Technology experimental results (Somers, 1997) and CFD results available in the literature, obtained for the same Reynolds number of $10^{6}$. The achieved good agreement suggests that the model has been validated for the steady flow regime as well. It is therefore suggested that the presented fluid-dynamic model might be a reliable mean to conduct both steady and unsteady analysis for high Reynolds numbers (fully turbulent flow regime).

\begin{tabular}{lll}
\hline & Experiments & CFD results \\
\hline$S t$ & 0.255 & 0.262 \\
$C_{l}[\mathrm{rms}]$ & 0.08 & 0.08 \\
$C_{d}[$ mean $]$ & 0.55 & 0.5 \\
\hline
\end{tabular}

Table 2: Smooth circular cylinder test validation with experiments data (ESDU, 1998).

\subsection{Strouhal number}

Two ranges of angles of attack have been considered, positive $\alpha=\left[20^{\circ} \rightarrow\right.$ $\left.160^{\circ}\right]$ and negative $\alpha=\left[-20^{\circ} \rightarrow-160^{\circ}\right]$. The ranges $\alpha=\left[0^{\circ} \rightarrow \pm 20^{\circ}\right]$ and $\alpha=\left[180^{\circ} \rightarrow \pm 20^{\circ}\right]$ have not been considered since at these angles the flow is largely attached and so no periodic vortex shedding would be expected. The CFD simulations have been run for a physical time of 10 s to allow the periodic wake associated with vortex shedding to become established. The transient initial period is discarded, and only established period vortex shedding is considered below.

Although large separated fluid regions are present in range of the ranges of $-30^{\circ}<\alpha<30^{\circ}$ and $150^{\circ}<\alpha<-160^{\circ}$, no evidence of periodic vortex shedding has been seen for these angles of attack, maybe due to the peculiarity of the simulation conditions. Therefore range of angle of attack discussed below can be confined to $\alpha=\left[40^{\circ} \rightarrow 140^{\circ}\right], \alpha=\left[-40^{\circ} \rightarrow-150^{\circ}\right]$.

A typical contour plot of instantaneous vorticity is shown in Figure 4. The particular case of $\alpha=50^{\circ}$ at 10 s is illustrated. The flow field immediately behind the airfoil, which is effectively a bluff body, is specific to the geometry. Close to the airfoil the wake structure appears to be highly asymmetric which 
is consistent with results from Perry and Steiner (1987) for a flat plate at an incidence angle of $45^{\circ}$. In the downstream wake a familiar vortex street can be seen.

The CFD simulations provide the temporal variation of the aerodynamic forces (lift, drag) and pitching moment. Figure 5 shows the time record of these aerodynamic coefficients for $\alpha=50^{\circ}$. The simulated time is $10 \mathrm{~s}$, giving then both the transient and the periodic part of the signal. For the sake of clarity, in Figure 6 the detail of these records with only $1.2 \mathrm{~s}$ of time is also presented. These plots are typical of what has been obtained for all the present simulations. It is apparent that all three quantities are periodic, with significant higher harmonics, leading to signals which are not pure sinusoids.

Figure 7 shows the Strouhal number trend against angle of attack. As expected, the frequency of the vortex shedding in the wake is a function of the angle of attack, consistent with what found by Nair and Sengupta (1997) in their experiments on elliptic cylinders at high angle of attack and moderate Reynolds numbers. Two normalisations are considered: the first uses the chord of the airfoil as the characteristic length $(d=c)$, while the other uses the projection of the chord on the vertical axis $(d=c \sin \alpha)$. The $S t$ trend in the case of the projected chord shows less variation than with the simple geometric reference length (as also can be seen used in Fage and Johansen, 1927).

No symmetry is present, with respect to the angular position of $\alpha= \pm 90^{\circ}$, due to the interchanging convex-concave as the upper-lower side of the airfoil. In general it can be noticed that the $S t$ takes higher values for the negative angles of attack compared with positive angles. This is probably due to the presence in negative angular positions of a cusp (i.e. trailing edge) on the leeward side of the airfoil which strongly affects the vorticity in the near proximity of the body.

\subsection{Mean loads coefficients}

The fluid loadings has been decomposed into mean and periodic components as shown in Eq.(2).

Figure 8 shows the variation of the mean forces and moment component coefficients $\left(C_{l 0}, C_{d 0}, C_{m 0}\right)$ with angle of attack. It can be seen that the lift coefficient changes in sign approaching $\alpha=90^{\circ}$. It is relatively high and is comparable to what would be expected at low angle of attack typical of the design operational range. However, unsurprisingly for high angles of attack, this is accompanied by a very high mean drag coefficient, which is 
one order of magnitude greater than what would be found at low angles. For a comparison, a flat plate at $\alpha=90^{\circ}$ presents a two-dimensional drag coefficient $C_{d} \approx 2$ (Simiu and Scanlan, 1978).

The moment behaviour instead is very flat.

There is no substantial difference in the mean coefficients between positive and negative angles of attack, indicating that the camber of the airfoil is not important at these high angles. Furthermore, the mean coefficients for angles in the range $\alpha=\left[40^{\circ} \rightarrow 90^{\circ}\right]$ and $\alpha=\left[-140^{\circ} \rightarrow-90^{\circ}\right]$ are comparable, indicating that the shape of the leading edge is unimportant as for $90^{\circ}<$ $\alpha<-90^{\circ}$ the sharp trailing edge is actually upwind of the rounded leading edge. These observations suggest that, at least for the mean force coefficients the values obtained here may be generally applicable to a wide range of airfoil sections.

\subsection{Fluctuating loads coefficients}

Figure 9 plots the variation with angle of attack of the coefficient of the lift force components for the first three harmonics of the vortex shedding frequency $\left(C_{l 1}, C_{l 2}, C_{l 3}\right)$. The main characteristic is that the first harmonic dominates, as would be expected, for the whole range of angles considered. The second harmonic is greater than the third. The trend of each harmonic for positive and negative angles is similar. However, it can be seen that for positive angles of attack the first harmonic of lift experiences a minimum around $70^{\circ}$, while for negative angles the trend is monotonic. This is due to the camber: geometrically, positive angles of attack present the oncoming flow with a concave shape, while negative angles present a convex profile. A more significant feature, in contrast to the mean coefficient, $C_{l 0}$, for angles where the sharp trailing edge is upwind $\left(90^{\circ}<\alpha<-90^{\circ}\right)$, the fluctuating lift force at the vortex shedding frequency is substantially higher, than when the rounded leading edge is up wind $\left(-90^{\circ}<\alpha<90^{\circ}\right)$. A peak can be seen in the second harmonic of lift for $|\alpha|=50^{\circ}$. It is not clear what causes this behaviour, hence further investigation is needed.

Figure 10 shows the variation with angle of attack of the coefficient of the drag force components for the first four harmonics of the vortex shedding frequency $\left(C_{d 1}, C_{d 2}, C_{d 3}, C_{d 4}\right)$. In this case, the even harmonics are as important as the odd harmonics, with the second harmonic dominating in the region around $\alpha= \pm 90^{\circ}$. This is consistent with vortex shedding for circular cylinder (Sonneville, 1976; Farell, 1981). The trends for all harmonics are similar for both positive and negative angles of attack, again suggesting 
that camber is unimportant. The coefficient for the second harmonic is lower when the sharp trailing edge is upwind, while the first harmonic is largely insensitive to this.

Figure 11 shows the associated coefficients for the pitching moment about the mid-chord for the first four harmonics $\left(C_{m 1}, C_{m 2}, C_{m 3}, C_{m 4}\right)$ which follow similar behaviour to the lift coefficient. In fact, as seen for the lift force, the first harmonic is dominating for the whole range of angles considered, though presenting higher values when the rounded leading edge is upwind $\left(-90^{\circ}<\alpha<90^{\circ}\right)$. Instead, the second harmonic is greater than the third just when the rounded leading edge is upwind $\left(-90^{\circ}<\alpha<90^{\circ}\right)$, and not in the whole range.

Figure 12 shows the phase angle relative to the lift coefficient of the dominant harmonic of the moment and drag coefficient, as determined in figures 9, 10 and 11. The subscript denotes the coefficients and the harmonic number. As can be seen the moment is always out of phase with the lift for all angles of attack. This is an artefact of the sign convention and the choice of the mid-chord for the centre of moment. The drag coefficient is a little harder to interpret. For $|\alpha|>120^{\circ}$ the first harmonic of the drag is dominant and so the phase difference is straight forward. For $|\alpha| \approx 90^{\circ}$ the second harmonic is the largest, so a pseudo phase angle is calculated as: $\phi_{l 1 d 2}=\phi_{l 1}-\frac{1}{2} \phi_{d 2}$. At a number of angles the first and second harmonics are comparable, and so it is not possible to extract a meaningful phase for these. What is apparent is that the phase of the drag is approximately 0 for negative angles and approximately $\pi$ for positive angles. This jump is effectively a minus sign in the amplitude which is related to the sign convention: a negative angle of attack will generate lift downwards, while drag is always in the stream-wise direction. The main thing to draw from this figure is that the moment and drag are synchronized with the lift. When the magnitude of lift increases due to a vortex being shed, so too does the moment and drag.

\section{Conclusions}

At high angles of attack a wind turbine blade section (NREL S809) will behave primarily as a bluff body causing vortex shedding and hence will experience fluctuating loads. In general, the wake shape is consistent with what is seen in the literature for common bluff bodies, suggesting that the vortex shedding process has been correctly captured. When the projected chord of the blade section is used to non-dimensionalise the vortex shedding 
frequency, the Strouhal number varies from 0.12 to 0.16 for negative angles $\alpha=\left[-40^{\circ} \rightarrow-150^{\circ}\right]$ and from 0.11 to 0.15 for positive angles $\alpha=\left[40^{\circ} \rightarrow\right.$ $\left.140^{\circ}\right]$.

The magnitude and harmonic content of the fluctuating forces and moment depend on the angle of attack. Furthermore, the strength of the loading depends on whether the sharp trailing edge is upstream or downstream; when the trailing edge is upstream, the first harmonic of lift and drag are higher whereas for the pitching moment is lower, but the second harmonic of drag is lower whereas for the pitching moment is higher. It has been observed that, as both positive and negative incidences provide similar behaviors in terms of periodic loads, the effect of the airfoil's chamber may be minimal. Therefore, the results obtained may be applicable to a wider range of airfoil's shapes.

Further work is required to assess the effect of three dimensionality caused by blade plan taper, low speed blade rotation or atmospheric boundary layer. Perhaps the most important unanswered issue is the effect of structural motion on the vortex shedding, particularly the lock-in range. Nonetheless, this current study represents an initial step towards a fully coupled model of vortex induced vibration of wind turbine blades.

\section{Acknowledgments}

This research is carried out under the EU FP7 ITN project SYSWIND (Grant No. PITN-GA-2009-238325). The SYSWIND project is funded by the Marie Curie Actions under the Seventh Framework Programme for Research and Technological Development of the EU. The authors are grateful for the support.

\section{References}

Anon, 2013. BP Statistical Review of World Energy 2013. BP.

Blevins, R., 1990. Flow-induced vibration.

ESDU, 1998. 96030 - Response of structures to vortex shedding: structures of circular or polygonal cross section, 1998. IHS (Global) Ltd.

Fage, A., Johansen, F., 1927. On the flow of air behind an inclined flat plate of infinite span. Proceedings of the Royal Society of London. Series A, 
Containing Papers of a Mathematical and Physical Character 116 (773), 170-197.

Farell, C., 1981. Flow around fixed circular cylinders: Fluctuating loads. Journal of the Engineering Mechanics Division 107 (3), 565-588.

Gerrard, J., 1966. The mechanics of the formation region of vortices behind bluff bodies. J. Fluid Mech 25 (2), 401-413.

IEC, 61400-1:2005+A1:2010. Wind turbines. Part 1: Design Requirements.

Medici, D., Alfredsson, P. H., 2006. Measurements on a wind turbine wake: 3d effects and bluff body vortex shedding. Wind Energy 9, 219 - 236.

Nair, M., Sengupta, T., 1996. Onset of asymmetry: flow past circular and elliptic cylinders. International Journal for Numerical Methods in Fluids 23 (12), 1327-1345.

Nair, M., Sengupta, T., 1997. Unsteady flow past elliptic cylinders. Journal of Fluids and Structures 11 (6), 555-595.

Paidoussis, M., Price, S., De Langre, E., 2010. Fluid-structure Interactions: Cross-flow-induced Instabilities. Cambridge Univ Pr.

Perry, A., Steiner, T., 1987. Large-scale vortex structures in turbulent wakes behind bluff bodies. part 1. vortex formation processes. J. Fluid Mech 174, 233-270.

Roshko, A., 1993. Perspectives on bluff body aerodynamics. Journal of Wind Engineering and Industrial Aerodynamics 49 (1-3), 79-100.

Sarpkaya, T., 2004. A critical review of the intrinsic nature of vortex-induced vibrations. Journal of Fluids and Structures 19 (4), 389-447.

Simiu, E., Scanlan, R., 1978. Wind effects on structures: an introduction to wind engineering. New York.

Somers, D.M., 1997. Design and experimental results for the S809 airfoil. Airfoils Inc., State College, PA, United States. NREL/SR-440-6918, ON: DE97000206, TRN: 97:001243. 
Sonneville, P., 1976. Etude de la structure tridimensionnelle des écoulements autour d'un cylindre circulaire. Bulletin de la Direction des Etudes et Recherches, Electricité de France, Serie A, No. 3.

Williamson, C., Govardhan, R., 2008. A brief review of recent results in vortex-induced vibrations. Journal of Wind Engineering and Industrial Aerodynamics 96 (6-7), 713-735. 


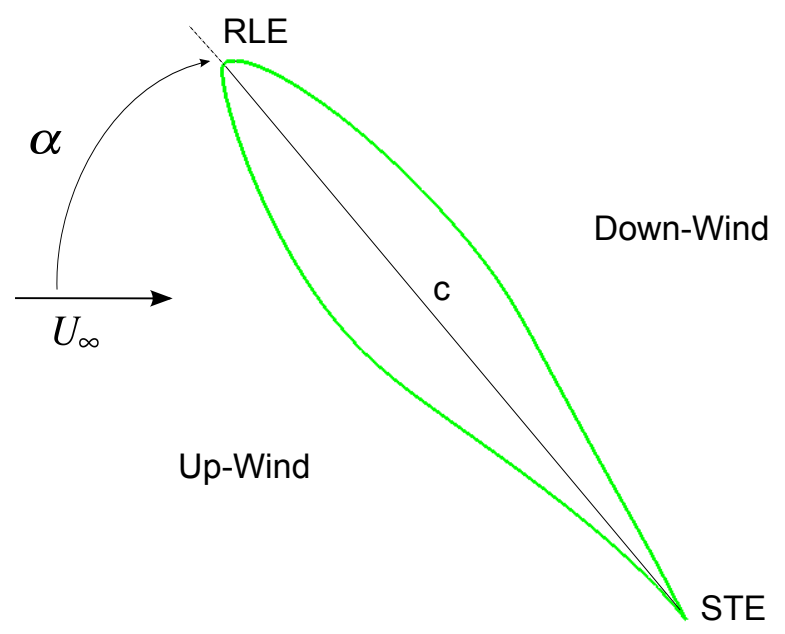

(a) $0^{\circ}<\alpha<90^{\circ}$,

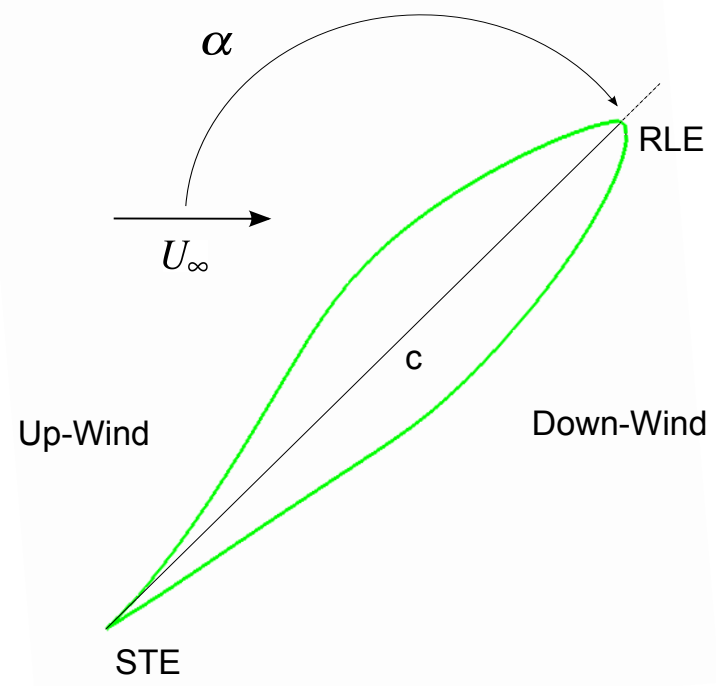

(b) $90^{\circ}<\alpha<180^{\circ}$.

Figure 1: Schematic of the NREL S809 airfoil for positive angles of attack. RLE is rounded-leading-edge, STE is sharp-trailing-edge. 


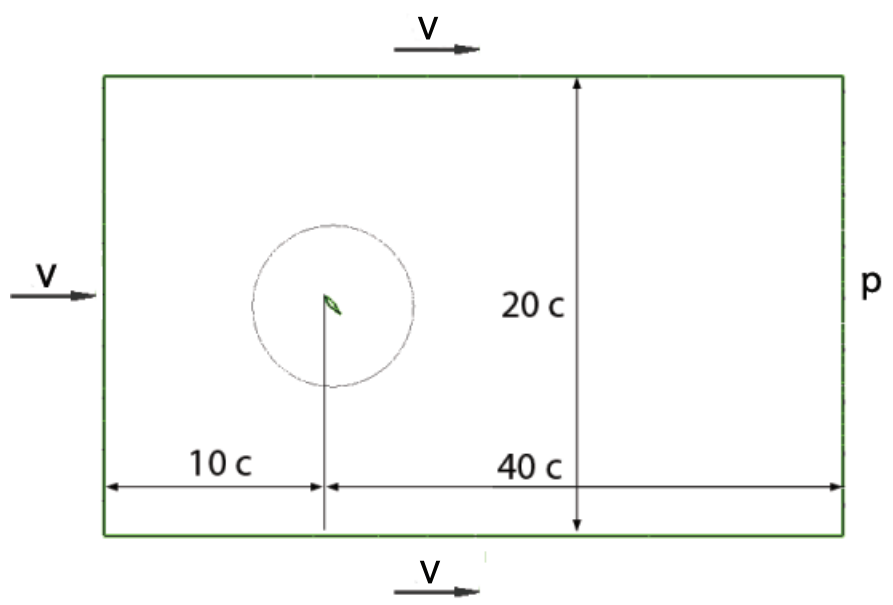

Figure 2: Flow field domain: dimensions (not in scale) and boundary conditions. 


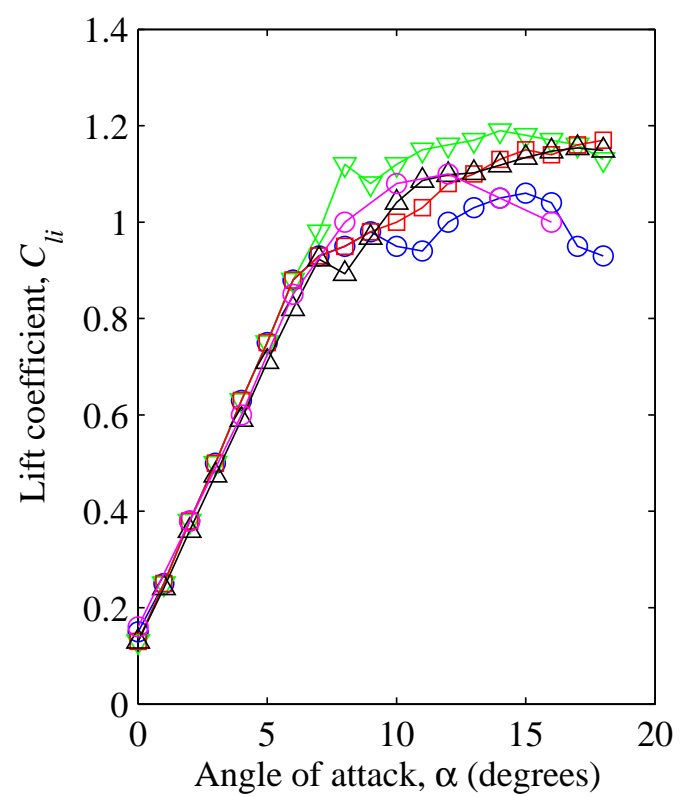

(a) Lift curve,

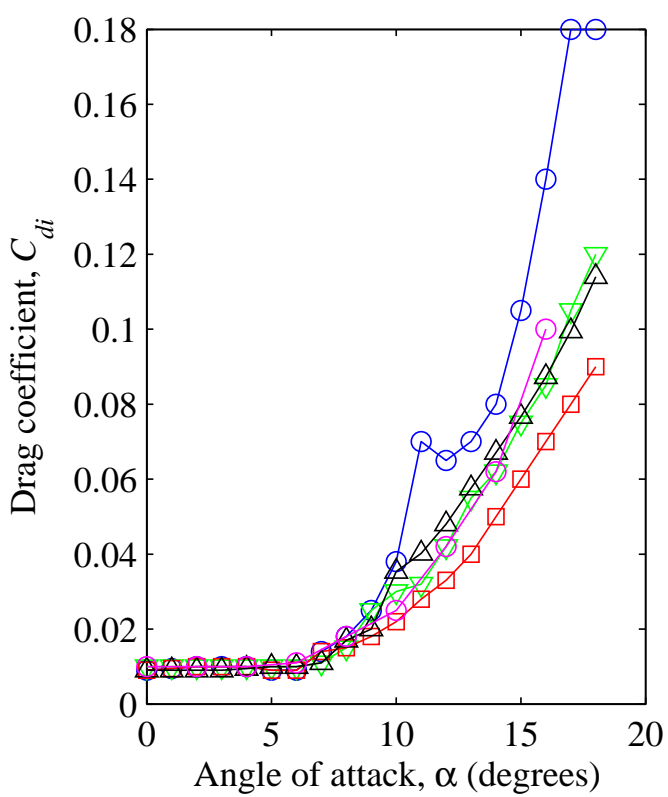

(b) Drag curve.

Figure 3: Steady aerodynamic force coefficients for the NREL S809 airfoil at $R e=10^{6}$ against angle of attack. Comparison between $-\bigcirc-$, Delft University Experiments (Somers, 1997) and CFD results: $-\nabla-$, ANSYSCFX; $-\square-$, XFOIL $6.0 ;-\bigcirc-$, ELLIPSYS $2 \mathrm{D} ;-\triangle-$, current study (ANSYS-Fluent). 


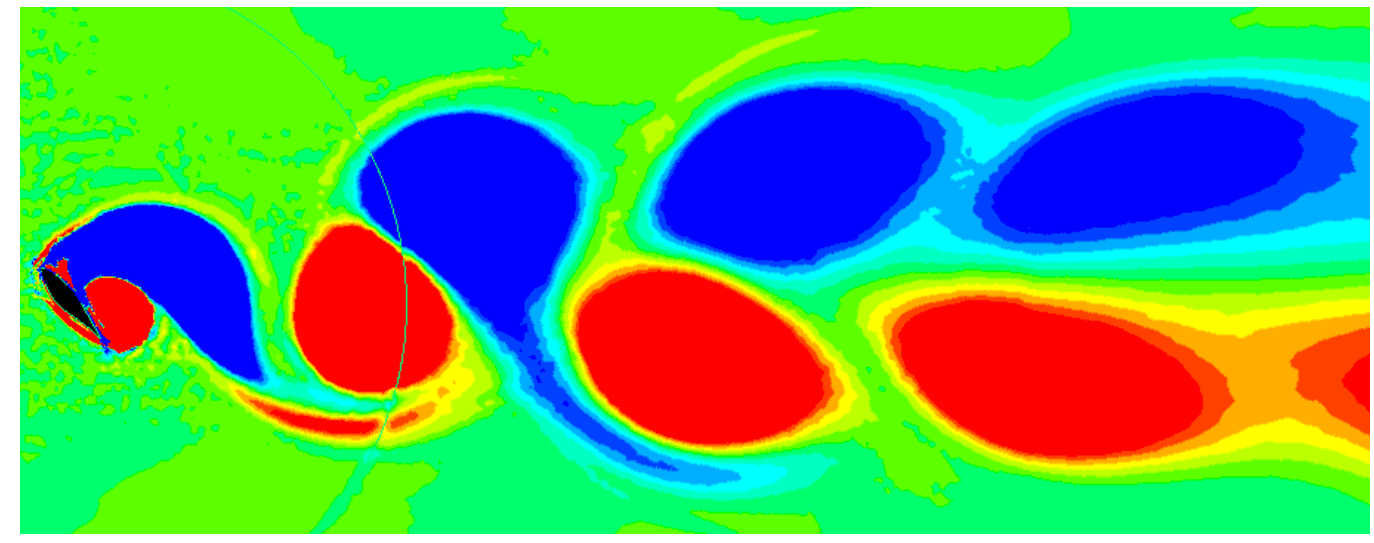

Figure 4: Instantaneous vorticity magnitude at $\alpha=50^{\circ}, \mathrm{t}=10 \mathrm{~s}$. Blue indicates clockwise direction, red counter-clockwise. 


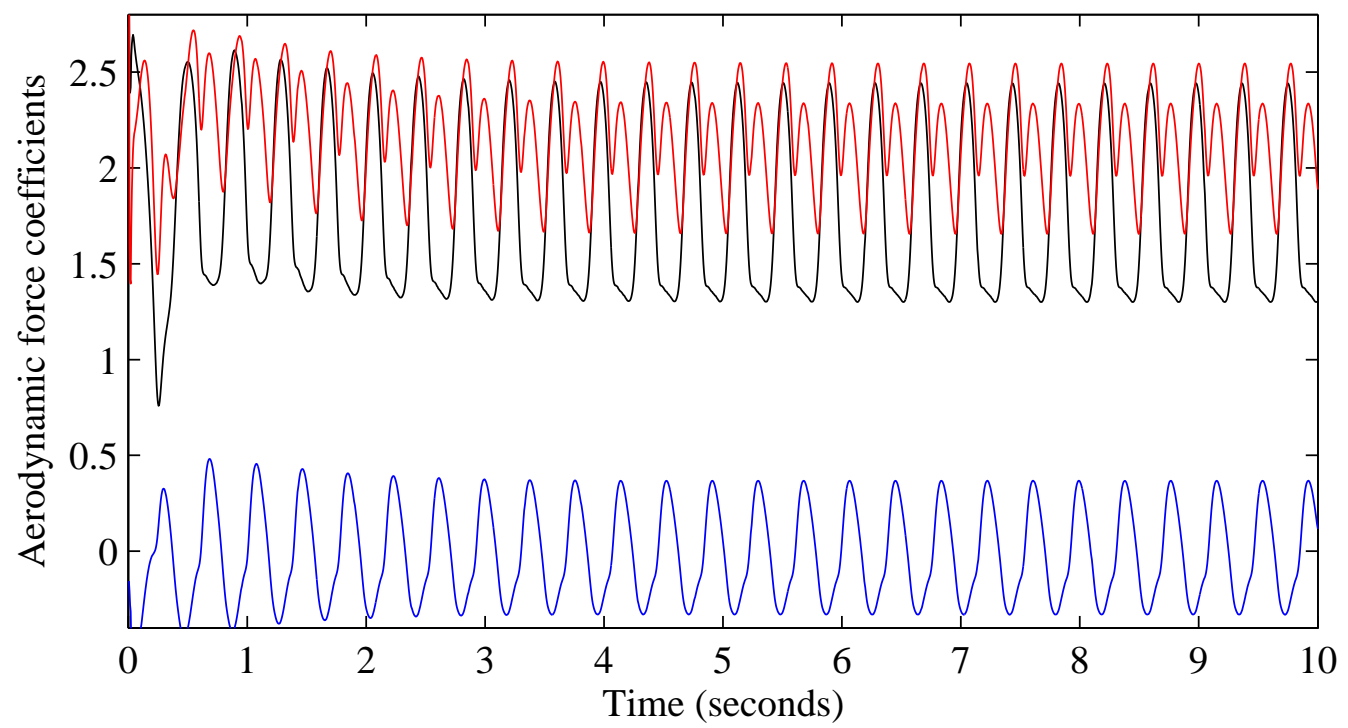

Figure 5: Time record of the aerodynamic force coefficients at $\alpha=50^{\circ}$ : lift coefficient, $C_{l}$; - , drag coefficient, $C_{d} ;-$, pitching moment coefficient, $C_{m}$. 


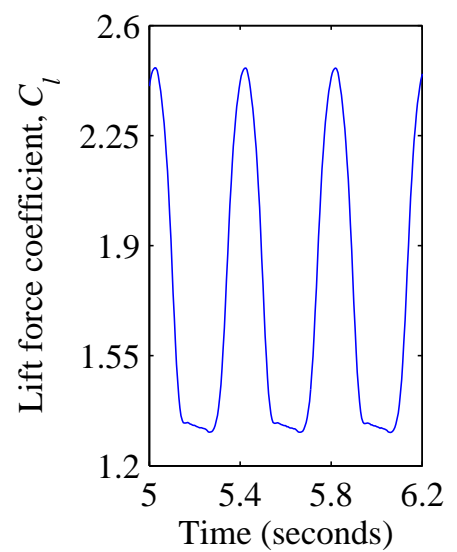

(a) Lift, $C_{l}$,

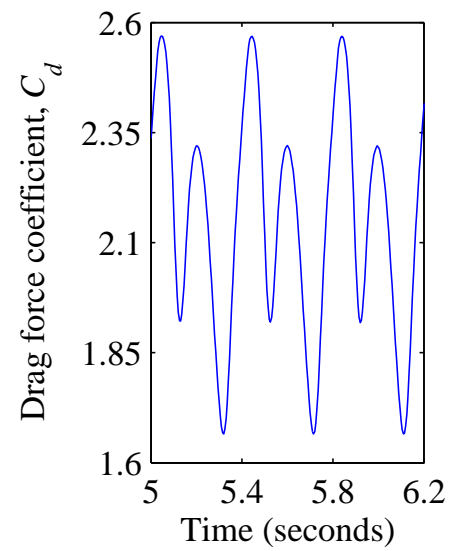

(b) Drag, $C_{d}$,

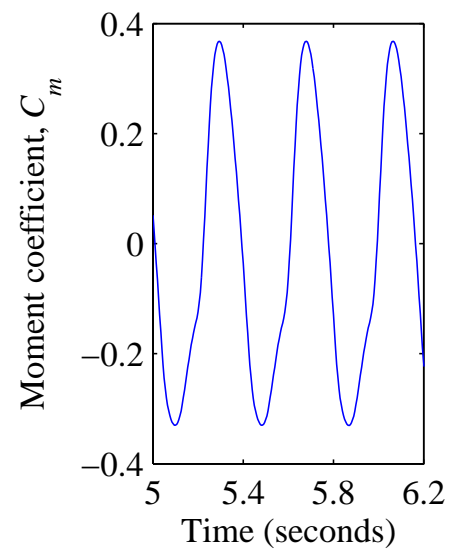

(c) Pitching moment, $C_{m}$.

Figure 6: Detail of the time record of the aerodynamic force coefficients at $\alpha=50^{\circ}$. 


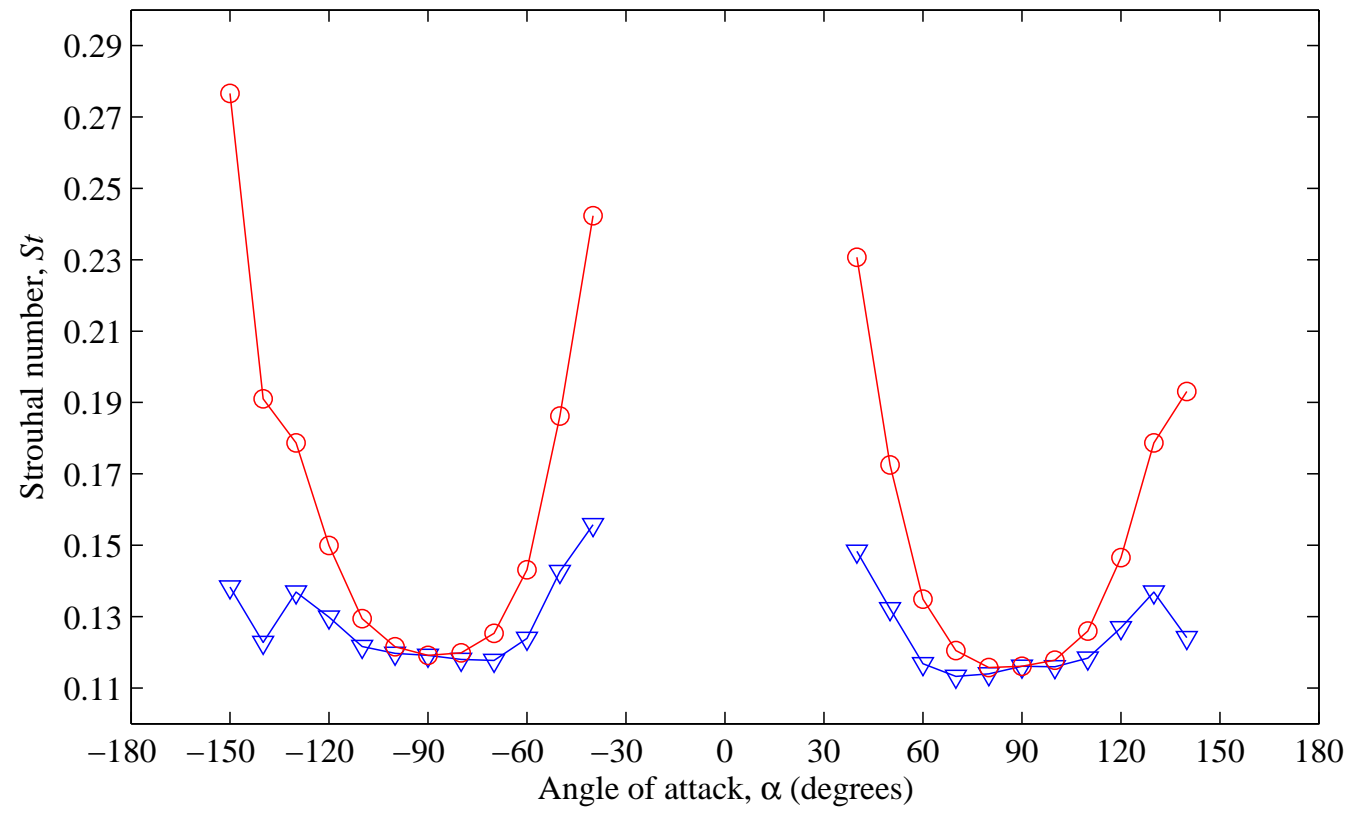

Figure 7: Strouhal number against angle of attack with different characteristic length: $-\bigcirc-$, airfoil chord $(c) ;-\nabla-$, projected chord $(c \sin \alpha)$. 


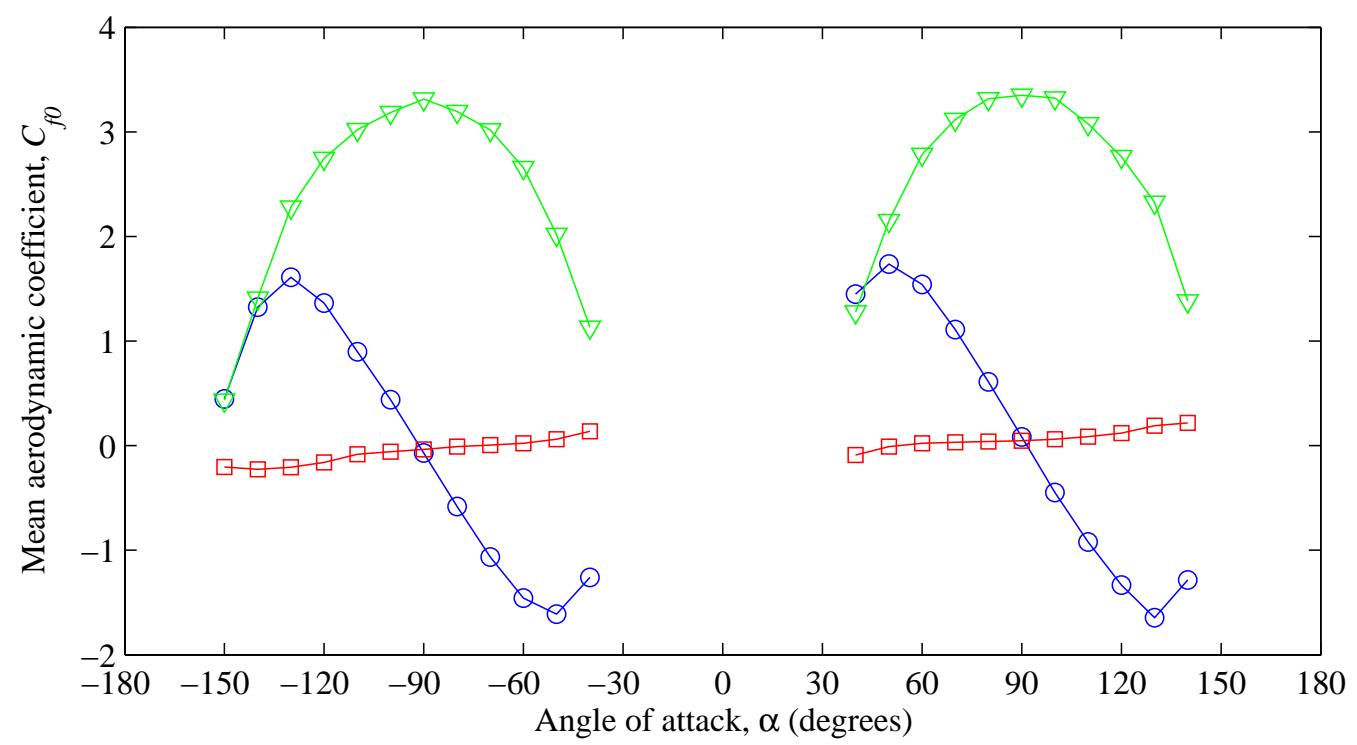

Figure 8: Mean forces component coefficients against angle of attack: $-\bigcirc-$, mean lift coefficient, $C_{l 0} ;-\nabla-$, mean drag coefficient, $C_{d 0} ;-\square-$, mean pitching moment coefficient, $C_{m 0}$. 


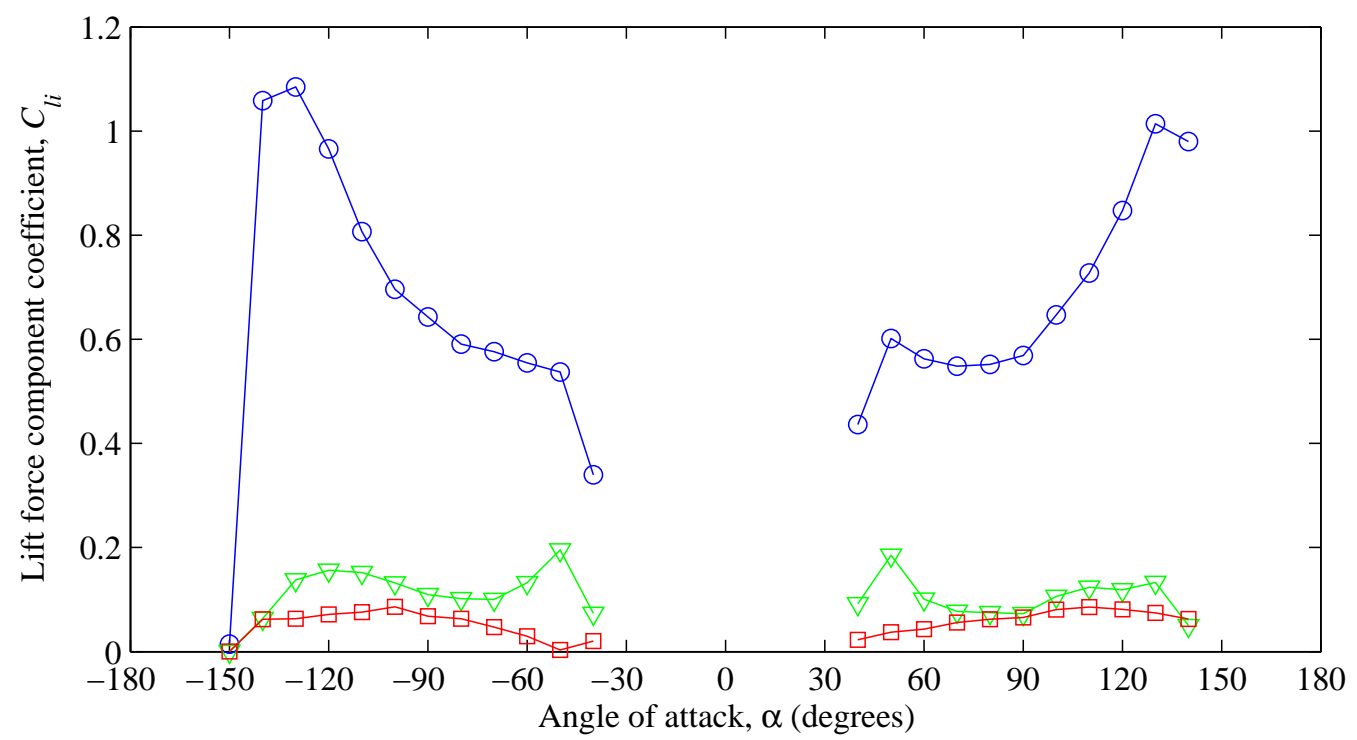

Figure 9: Lift force component coefficients against angle of attack: $-\bigcirc-$, first harmonic; $-\nabla-$, second harmonic; $-\square-$, third harmonic. 


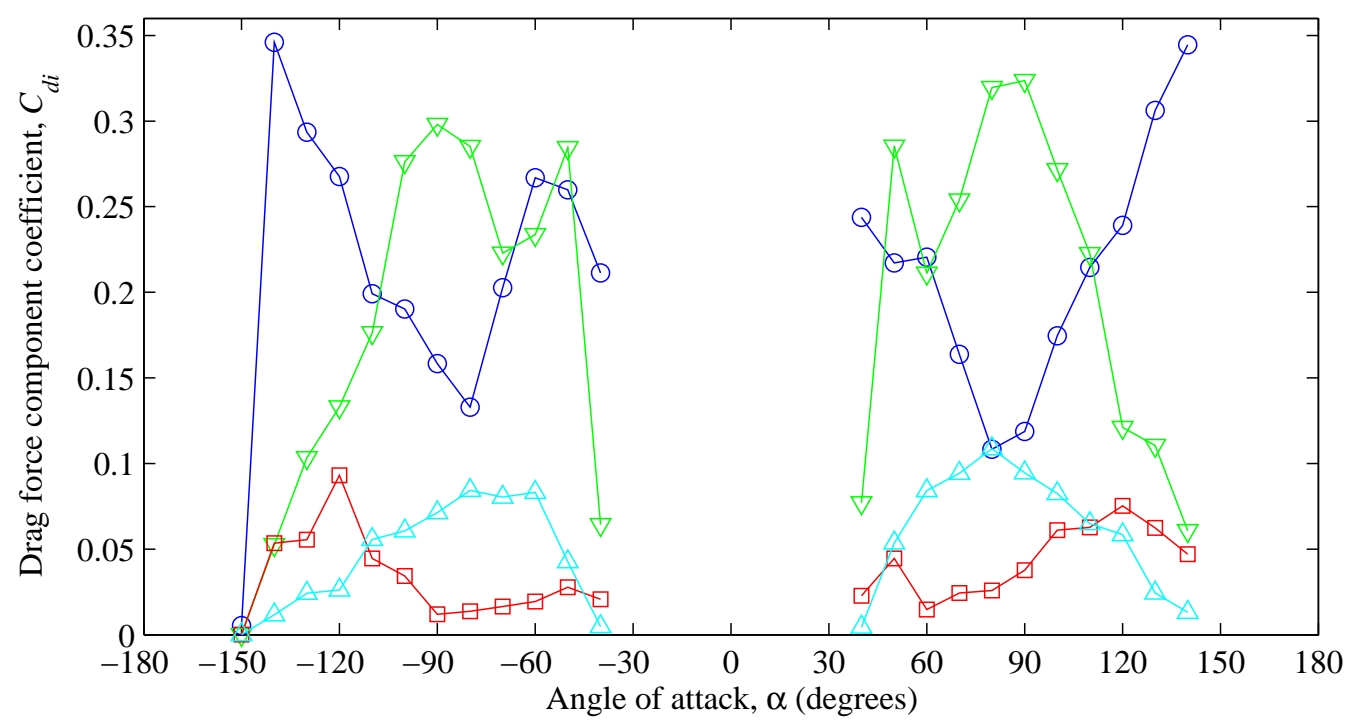

Figure 10: Drag force component coefficients against angle of attack: $-\bigcirc-$, first harmonic; $-\nabla-$, second harmonic; $-\square-$, third harmonic; $-\triangle-$, fourth harmonic. 


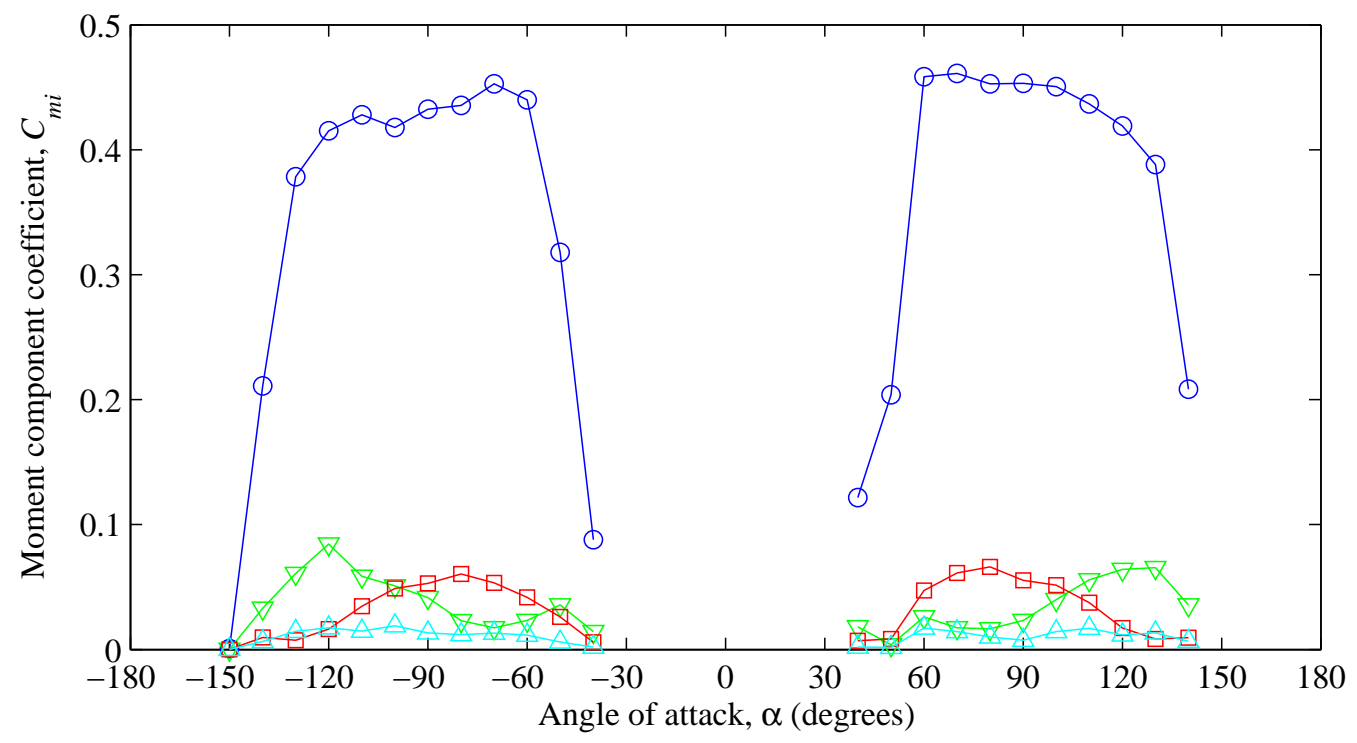

Figure 11: Moment component coefficients against angle of attack: - $\bigcirc-$, first harmonic; $-\nabla-$, second harmonic; $-\square-$, third harmonic; $-\triangle-$, fourth harmonic. 


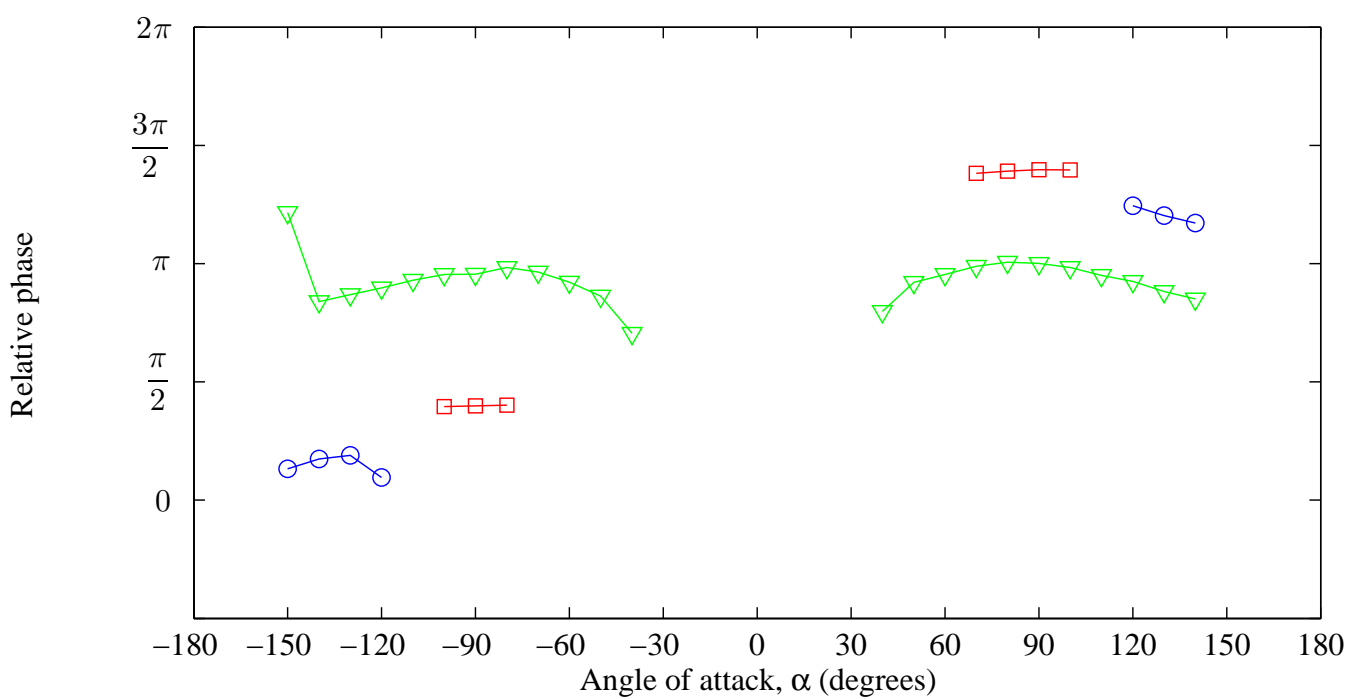

Figure 12: Relative phase of dominant harmonic: $-\bigcirc-, \phi_{l 1 d 1} ;-\square-, \phi_{l 1 d 2}$; $-\nabla-, \phi_{l 1 m 1}$. 